\title{
Characterization of Outpatients With Isolated Diastolic Dysfunction and Evaluation of the Burden in a Japanese Community Sado Heart Failure Study
}

\author{
Yuji Okura, MD ${ }^{\dagger \dagger}$; Yukiko Ohno, MD ${ }^{\dagger \dagger}$; Mahmoud M Ramadan, MD; Keisuke Suzuki, MD*; \\ Koji Taneda, MD*; Hiroaki Obata, MD; Komei Tanaka, MD; Takeshi Kashimura, MD; \\ Osamu Ishizuka, MD**; Kiminori Kato, MD; Haruo Hanawa, MD; \\ Yasuyuki Honda, $\mathrm{MD}^{\dagger}$; Makoto Kodama, MD; Yoshifusa Aizawa, MD
}

\begin{abstract}
Background The incidence of diastolic heart failure (DHF) is increasing with the aging of the community and identifying patients with isolated diastolic dysfunction (IDD) is important for preventing DHF. However, very little information is available about such patients in the Japanese community.

Methods and Results The medical information of all outpatients with moderate to severe IDD was extracted from the records of approximately 6,948 individuals who underwent echocardiographic (Echo) examinations during the past 5 years in Sado Island. Of the 284 patients extracted, 272 survived until 2003. In January 2003 the proportion of patients with moderate to severe IDD in the general population sector aged 45-84 years was $0.9 \%$ for males and $0.5 \%$ for females, and this proportion increased sharply after the age of 65 in both genders, reaching $1.6 \%$ for men in their $70 \mathrm{~s}$ and $0.8 \%$ for women in their $80 \mathrm{~s}$. On Echo, 165 patients $(61 \%)$ showed hypertrophic left ventricular geometry. The Charlson comorbidity index score was $\leq 1$ in $63 \%$ of patients. The cumulative survival of IDD patients, irrespective of a history of congestive heart failure (HF), was significantly lower than in the general population.

Conclusions Moderate to severe IDD is not uncommon in the elderly and has a poor prognosis. Characteristics of outpatients with IDD should be taken into consideration when establishing a preventive strategy for HF in the Japanese community. (Circ J 2007; 71: 1013-1021)
\end{abstract}

Key Words: Diastolic dysfunction; Disease burden; Charlson comorbidity index; Heart failure

$\mathbf{T}$ he prevalence of heart failure (HF) is increasing progressively in developed countries with the aging of the population! Recently, some studies have reported a large number of individuals with isolated diastolic dysfunction (IDD) in the general population, ${ }^{2,3}$ The clinical presentation of IDD is very diverse, ranging from no symptoms to so-called diastolic HF (DHF), which manifests clinically as congestive HF (CHF). IDD is a new target of therapy for the prevention of HF. Compared with the diagnosis of left ventricular (LV) systolic dysfunction (SD), the diagnosis of IDD is rather more complicated and easily overlooked.

Management of IDD may be important for reducing the future burden of HF in Asian communities, where aging of the populating is rapidly advancing. Not a few patients are

(Received January 23, 2007; revised manuscript received April 12, 2007; accepted April 17, 2007)

Division of Cardiology, First Department of Medicine, Niigata University Graduate School of Medical and Dental Sciences, Niigata,

*Sado General Hospital, **Ryotsu Hospital and Honda Clinic, Sado, Japan

$\dagger \dagger$ The first and second authors contributed equally to this work.

Mailing address: Yuji Okura, MD, Division of Cardiology, Niigata

University Graduate School of Medical and Dental Sciences, 1-754

Asahimachi, Niigata 951-8510, Japan. E-mail: okuray@med.niigata-u. ac.jp presumed to be under treatment for IDD, but information is scarse. Therefore, to delineate the epidemiological and clinical features of patients with IDD, we carried out complete enumeration of them in Sado City, via co-operation with the core hospitals and medical societies.

\section{Methods}

Selection of Cases

The Sado Heart Failure Study was designed to characterize outpatients with cardiac dysfunction at the hospitals and clinics of Sado City, an island city in Japan. Within a total period of 5 years (extending from January $1^{\text {st }} 1998$ till December $31^{\text {st }} 2002$ ), patients with IDD were extracted from the records of 6,948 consecutive individuals who had their echocardiographic (Echo) examinations performed at 2 core hospitals in Sado City. In case the same patient was extracted twice or more during the recruitment period, only the data for the first extraction was considered.

\section{Definition of IDD}

IDD was defined as LV ejection fraction (LVEF) $\geq 40 \%$, plus at least 1 of the following criteria (after excluding moderate or severe mitral and aortic insufficiency): (1) deceleration time (DCT) <140 ms; (2) ratio between systolic (S) and early diastolic (D) waves of the antegrade flow of the pul- 
Table 1 Charlson Comorbidity Index ${ }^{7,8}$

\begin{tabular}{l}
\hline \hline Disease \\
\hline Myocardial infarction \\
Congestive heart failurex \\
Peripheral vascular disease (arteriosclerosis obliterans, acute arterial occlusion) \\
Dementia \\
Chronic pulmonary disease \\
Connective tissue disease \\
Mild liver disease (chronic viral hepatitis) \\
Diabetes mellitus \\
Hemiplegia \\
Moderate or severe renal disease (serum creatinine $\geq 2$ mg/dl or hemodialysis) \\
Any tumor \\
Leukemia \\
Lymphoma \\
Moderate or severe liver disease (fulminant hepatitis, esophageal varices, liver cirrhosis) \\
Metastatic solid tumor
\end{tabular}

monary venous flow velocity tracing $(\mathrm{S} / \mathrm{D}$ ratio $)<1$; and (3) difference between the duration of pulmonary venous atrial reversal flow and transmitral flow at atrial contraction tracing (literally AR-A) $>30 \mathrm{~ms}$.

These criteria have been used to identify moderate (pseudonormal) or severe (restrictive) diastolic dysfunction (DD) in other studies, ${ }^{2}, 3$ In order to reduce oversight and include suspected cases, we included any patient who fulfilled at least 1 of the criteria. The possession of the first criterion was necessary to include patients with chronic atrial fibrillation (AF). Patients with LVEF $<40 \%$ were counted and grouped under the SD category, whereas those with LVEF < $50 \%$ (with none of the abovementioned criteria) were considered as the control group for IDD patients for comparative purposes.

\section{Review of Cases}

All medical records of the extracted patients were reviewed, and their clinical data were collected and summarized according to a predetermined protocol. If a patient consulted 2 or more medical institutions during the recruitment period, as far as possible all the records at those institutions were reviewed.

\section{Extraction of Patients' Data}

The available clinical records were reviewed with regard to demography, medical history, comorbidities, medical therapies, laboratory investigations, electrocardiographic (ECG) and Echo examinations. LV hypertrophy (LVH) was defined as high voltage [Sokolow-Lyon voltage (SV1+ $\mathrm{RV} 5 / 6)>38 \mathrm{~mm}$ ] associated with left atrial enlargement and/or ST-T change in the left precordial leads of the ECG. Echocardiographic LV mass (g) was calculated according to the method recommended by the American Society of Echocardiography $[\mathrm{LV}$ mass $=0.8 \times(1.04 \times[\mathrm{LVDd}+\mathrm{IVST}+$ PWT] $\left.\left.{ }^{3}-\mathrm{LVDd}^{3}\right)+0.6\right]$ and then indexed to body surface area $\left(\mathrm{m}^{2}\right)$ (LVDd, LV diastolic dimension; IVST, interventricular septal thickness; PWT, posterior wall thickness).5 In the patients who survived, the information was collected up to January $1^{\text {st }} 2003$. Present or past history of CHF was confirmed using the Framingham criteria6 In addition, we evaluated the effects of comorbidities (included or not in the Charlson comorbidity index (CCI)) on patients' survi$\mathrm{val}^{7,8}$ (Table 1). Although the CCI is a survival index, it has also been validated to correlate with physical disabilities and re-admission rates in patients with chronic HF8,9

\section{Confirmation of Survival}

Confirming the survival of patients with available hospital records up to or beyond January $1^{\text {st }} 2003$ was quite easy; but for those without such records, direct phone calls to their families or attending physicians were made. Based on the available clinical information, professional cardiologists classified the causes of death as cardiac or noncardiac, according to previous epidemiological studies conducted in Japan!0,11 Cardiac death was defined as death from cardiac events: fatal myocardial infarction (MI), HF, or sudden cardiac death. Noncardiac death was defined as death from noncardiac causes (such as cancer).

\section{Determination of the Proportion of IDD Patients in the General Population}

This was done using the following equation:

$\frac{\text { No. of DD survivors up to January } 1^{\text {st }} 2003}{\text { Estimated population of Sado City on January } 1^{\text {st }} 2003} \times 100$

The numerator estimate represents the Echo-documented IDD patients extracted for the whole period of this study (5 years) after excluding those in whom survival status was impossible to be determined. The denominator estimate was obtained from the Niigata Prefectural Department of Policy and Planning, Niigata, Japan!2 The obtained proportion was compared after stratification by gender and 5-year age intervals.

\section{Survival Analysis}

Survival curves of the general population of Sado City were prepared for both genders on the basis of the Niigata Prefecture Life Table (2000) using actuarial analysis! $!^{13,14}$ The latter table was made according to the National Life Table, which is based on the results of the Japan Population Census (2000). Similarly, survival curves of patients with IDD were prepared, stratified by gender, and compared with the general population curves by log-rank test. Each of these curves was drawn by linking the points representing the average survival rate of each 5-year age group. We excluded patients with malignant conditions $(n=34)$, because they may have an impact on the effect of IDD on survival.

\section{Statistical Analysis}

Data analyses were performed with SPSS for Windows Version 13 (SPSS Inc, Chicago, IL, USA). Results for con- 
Patients* with diastolic dysfunction (DD) recruited from 1998 to $2002(\mathrm{n}=394)$

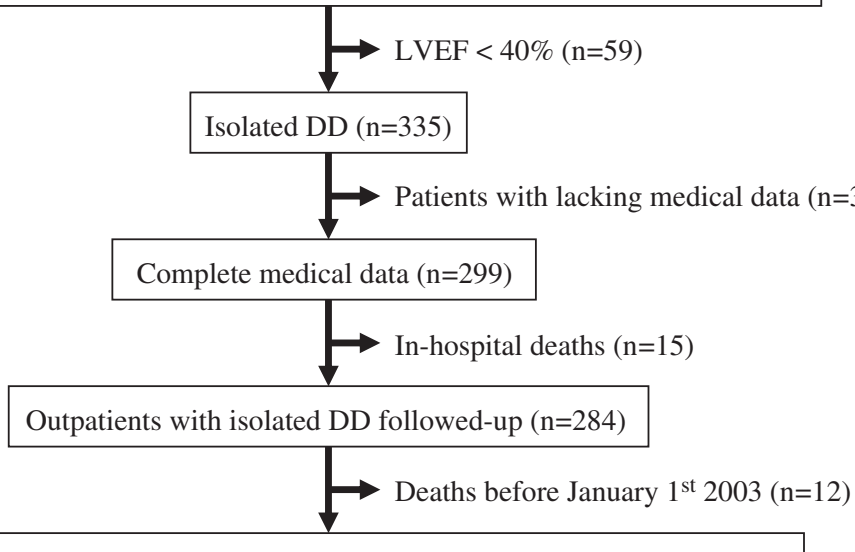

Survivors until January $1^{\text {st }} 2003$ eligible for inclusion in our study $(n=272)$
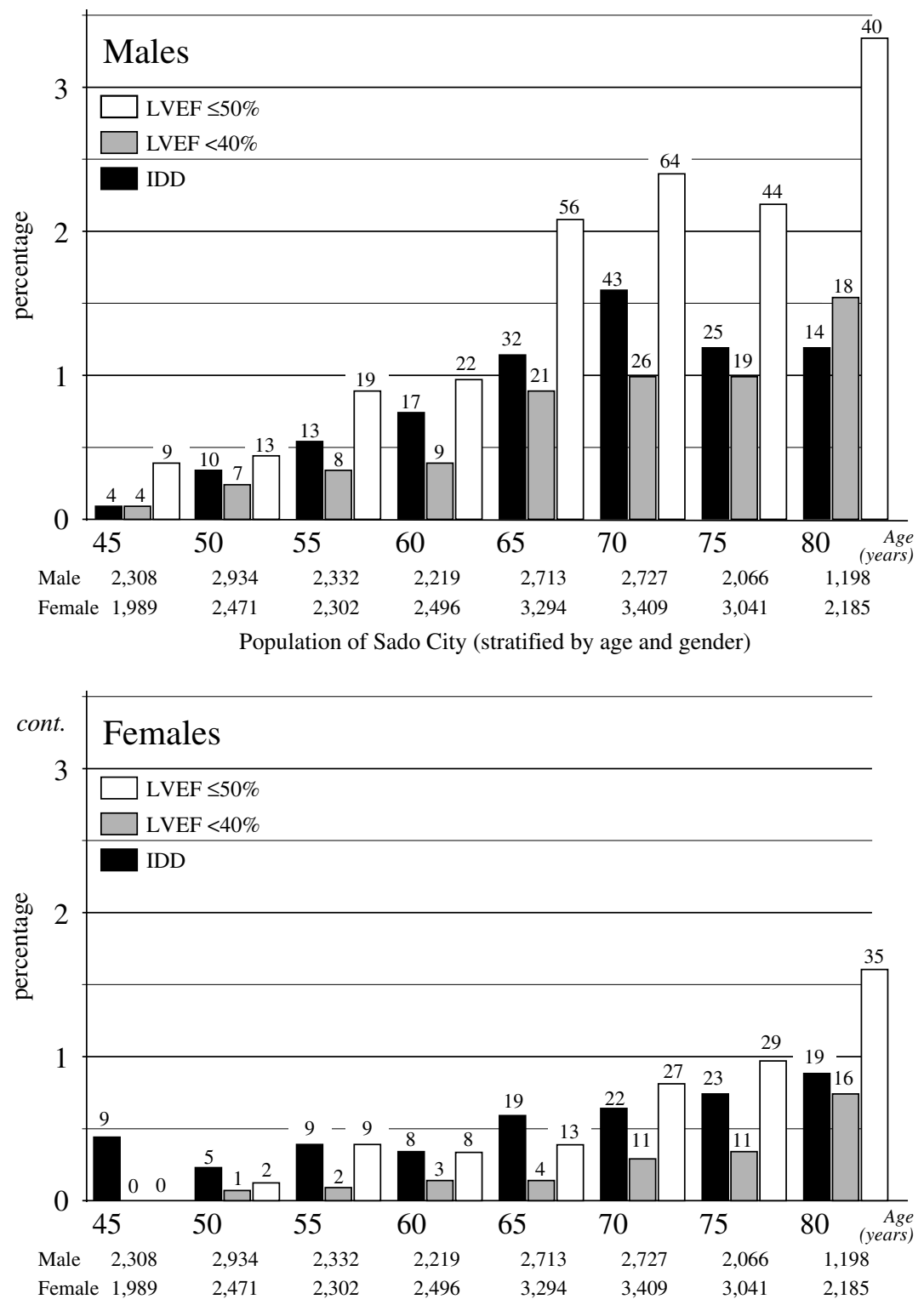

Population of Sado City (stratified by age and gender)
Fig 1. Flow chart of Sado Heart Failure study, showing the process of extraction and followup of patients with isolated diastolic dysfunction (IDD). The starting date of the recruitment period was set at 5 years (from January $1^{\text {st }} 1998$ until December 31st 2002), and 335 patients were extracted. Out of this number, 63 patients were excluded due to a lack of medical records, unavailable diagnosis, in-hospital (pre-discharge) mortality, or death on follow-up. Thus, 272 patients finally survived until January $1^{\text {st }}$ 2003 and were included in the calculation of the prevalence of IDD. *Residents of Sado City with expected age range $45-48$ years on January $1^{\text {st }} 2003$.
Fig 2. Bar graphs showing the proportion of isolated diastolic dysfunction (IDD) and systolic dysfunction (SD) patients in the general population for males and females on January 1 st 2003 . The number shown at the upper end of each bar represents patients' count, while the numbers below each age range along the horizontal axis represent the male and female populations for that range. LVEF, left ventricular ejection fraction. 


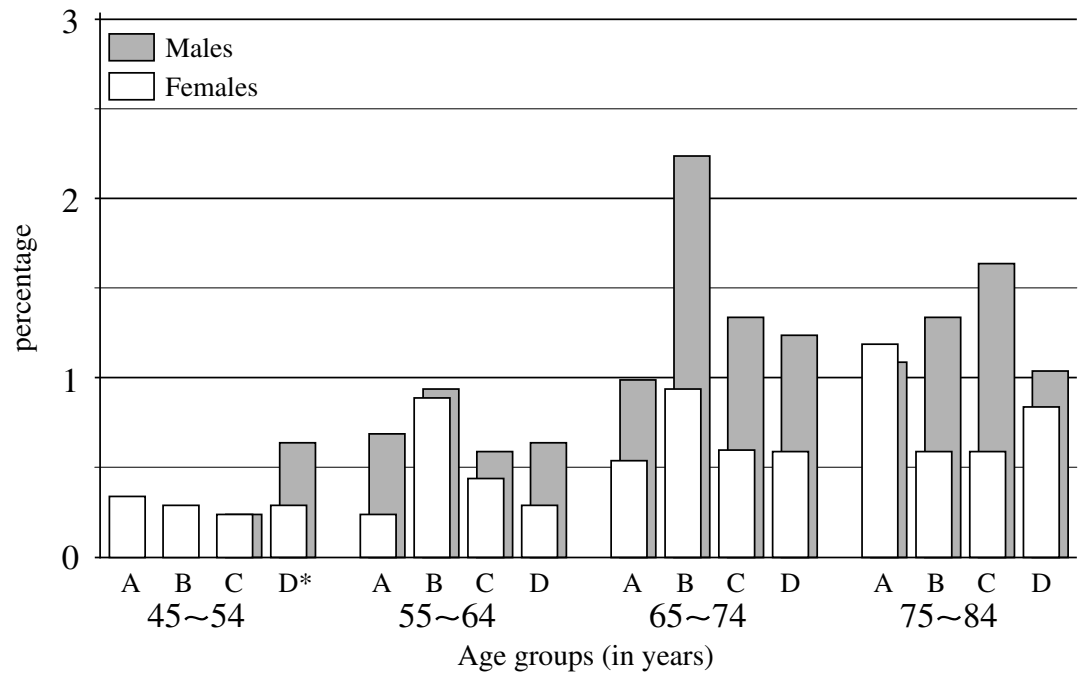

Fig 3. Bar graphs showing the regional proportion of isolated diastolic dysfunction (IDD) patients in the general population of Sado City on January 1 1st $^{\text {s. }} 2003$ (classified by gender and age groups). No significant difference regarding the proportion of IDD was observed among the 4 regions in any age group for either gender. *The letters A to D represent the 4 divisions of Sado Island. (a)

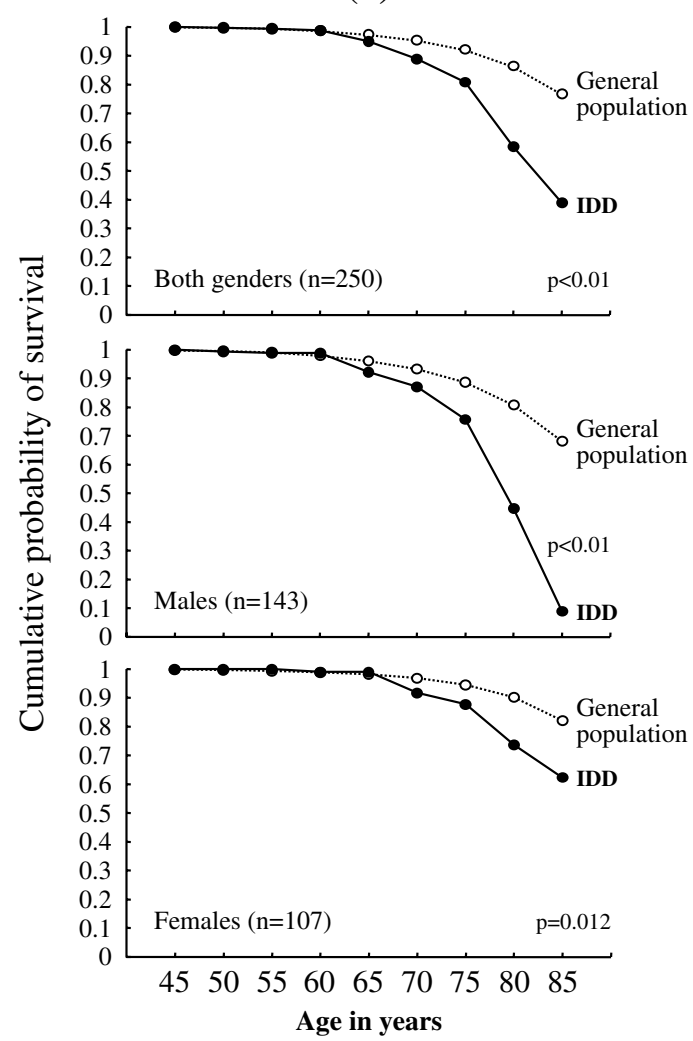

(b)

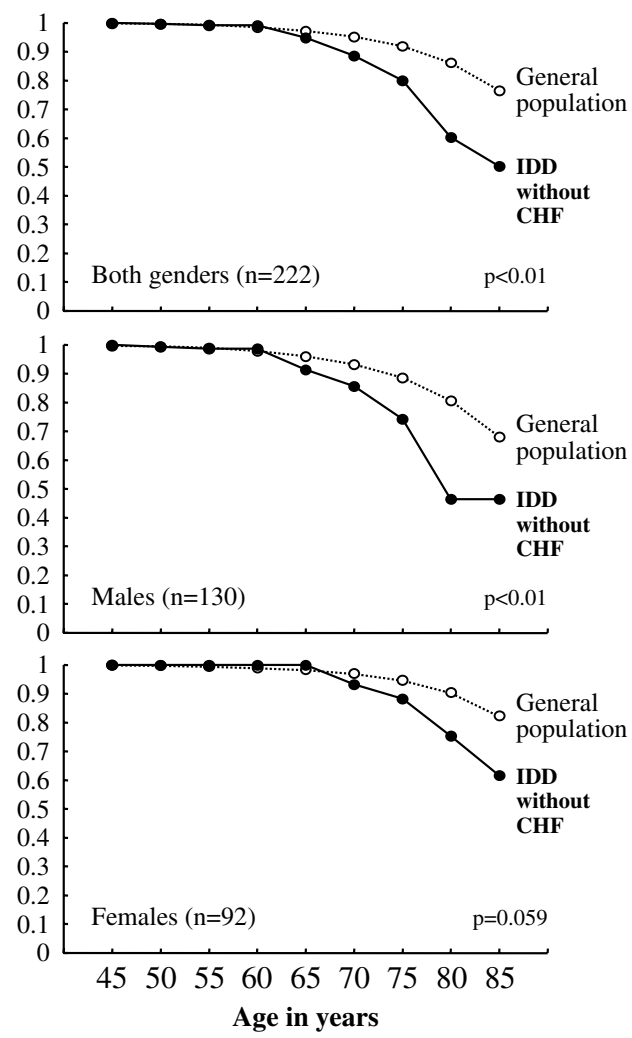

Fig 4. Comparative analysis of survival between patients with isolated diastolic dysfunction (IDD) and the general population. Survival curves of the general population (on the basis of Niigata Prefecture Life Table [2000]), and those of patients with IDD (a) as well as those of IDD without history of congestive heart failure (CHF) (b) were prepared by using actuarial methods; and comparisons were made by log rank test. Compared to the general population, the survival rate was significantly lower in patients with IDD and IDD without history of CHF. IDD without CHF, IDD without history of CHF.

tinuous normal data are expressed as mean \pm standard deviation. Comparison of means of continuous variables across a grouping variable with 2 levels was done by Student's t-test. Comparison of proportions was made by ${ }^{2}$ test with Yates's correction. A 2-sided significance level of 0.05 was used for all analyses.

\section{Ethical Considerations}

The study protocol was reviewed and accepted by the local Ethical Review Board of each medical institution participating in this study, including the Niigata University Graduate School of Medical and Dental Sciences, and written consent was given by all patients. 


\section{Results}

Follow-up of Extracted Patients (Fig 1)

A total of 394 patients with DD were extracted within the 5-year recruitment period (from January $1^{\text {st }} 1998$ till De-

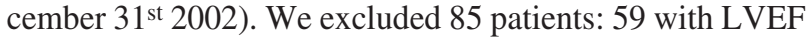
$<40 \%$, and 36 who either lacked medical records or we were unable to confirm their survival. Moreover, 15 in-hospital deaths (before discharge) were excluded, as well as 12 deaths that, on careful follow-up, were ascertained to take place before January $1^{\text {st }}$ 2003. Thus, 272 patients $(58 \%$ males) were confirmed to have survived up to that date and were eventually included in the study.

\section{Proportion of IDD Patients in the General Population}

in Relation to Age, Gender, and Region

The proportion of IDD patients in the general population sector aged $45-84$ years was $0.85 \%$ for males and $0.54 \%$ for females (Fig 2). This proportion increased sharply after the age of 65 in both genders, reaching $1.6 \%$ for men in their $70 \mathrm{~s}$ and $0.8 \%$ for women in their $80 \mathrm{~s}$.

The proportion of IDD in the 4 zones of Sado Island was compared after stratification by gender and age decades (Fig 3). No significant difference in the proportion of IDD was found in either gender within any age group among the 4 areas.

\section{Comparison of Patients' Survival Rates Relative to the General Population}

We followed 284 outpatients with IDD from diagnosis

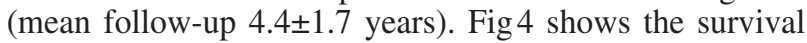
curves of the general population (based on the life table) compared with those of the IDD patients without a history of malignancies $(n=250)$, before and after stratification by gender. In comparison with the general population, the IDD patients cumulative survival rate was significantly lower for both males and females. On the other hand, in patients with IDD without CHF history, the survival of males was significantly lower than that of the general population; females did not exhibit such a significant difference.

\section{Characteristics of IDD Patients}

Associated Comorbidities Adequately documented present or past histories of comorbidities are shown for both genders in Table 2. Hypertension was the most prevalent comorbidity $(46 \%)$ for both genders. Traditional cardiovascular risk factors were also prevalent among these patients; nearly one-quarter (24\%) were diabetic, and dyslipidemia and abnormal body weight were found in $22 \%$ and $19 \%$ of cases, respectively. Twenty-eight percent of all patients underwent cardiac catheterization, and ischemic heart disease was frequently observed in males (19\%) and females $(15 \%)$. AF was the second most prevalent comorbidity associated with IDD in all cases (33\%) and in either gender. Of 206 patients with normal sinus rhythm in 2003, 25 (12\%) of them had a history of AF (ie, paroxysmal AF). Notably, a considerable percentage $(21 \%)$ of patients had had a cerebral stroke, and psychiatric disorders, such as depression and dementia, were found in $11 \%$ of all cases (significantly more prevalent in females).

Level of Daily Activities and Lifestyle The majority of patients (93\%) were independently mobile (Table2) and wheel chair-dependents actually constituted a minority (3\%). Notably, women tended to lead a solitary life more often than men who tended to live in families.
Medications Approximately $41 \%$ of patients were on calcium-channel blocker blockers (CCBs), and 39\% were taking angiotensin-converting enzyme inhibitors or angiotensin-II receptor blockers (Table 2). Diuretics were given to $28 \%$ of patients, and a considerable percentage of cases were being maintained on anticoagulant (28\%) and antiplatelet $(23 \%)$ therapy.

ECG Findings As shown in Table 2, the most frequent abnormality found in patients with IDD was AF (24\%) followed by LVH (17\%); none of them had left bundle branch block.

Echo Findings Left atrial enlargement was observed in males and females with IDD, and LVEF was in the normal range. Various LV geometric patterns ${ }^{5}$ were carefully evaluated in these patients. Hypertrophic geometry, such as eccentric or concentric hypertrophy, was the most frequent pattern ( $54 \%$ of males, $69 \%$ of females), followed by normal LV geometry (34\% of males, $28 \%$ of females). Concentric remodeling was rarely found in either gender (only $8 \%$ of all cases). Regarding the Doppler Echo findings, DCT $<140 \mathrm{~ms}, \mathrm{~S} / \mathrm{D}$ ratio $<1$, and AR-A duration $>30 \mathrm{~ms}$ were observed in $45 \%, 51 \%$ and $29 \%$ of patients, respectively. The isovolumetric relaxation time (IRT) was shortened in both genders compared with the normal average. Apart from the S/D ratio, none of the Doppler-Echo parameters exhibited a significant association with gender.

Functional and Prognostic Indices Cardiac function was controlled at New York Heart Association class II or III in $58 \%$ of patients, but a minority (1\%, all were males) progressed to the gravest class IV (Table 2). For the CCI, a score of 1 point is given for each of the following conditions: CHF, MI, and diabetes (Table 1). Thus, approximately $36 \%$ of IDD patients were free of comorbidities (a score of 0 ), and nearly equal proportions (28\% and $25 \%$ ) had scores of 1 and $2-3$, respectively. Only $11 \%$ of patients had a score $\geq 4$, indicating a low possibility of survival?

Predictors of Mortality Mortality was reported in 50 patients $(6.3 \%)$ on follow-up: $12(24 \%)$ from cardiac causes and $30(60 \%)$ from noncardiac reasons (11 strokes, 11 deaths from cancer, 8 for other reasons). The cause of death was unknown in 8 cases (16\%). A Cox proportionate hazard regression model was constructed for identifying the independent predictors of death for each gender. Simple univariate analysis was done first for each of the input variables against positive death outcome, and all the variables associated with it at $p \leq 0.15$ were finally entered in a multivariate forward stepwise Cox regression model. The correlation matrix was carefully checked to avoid collinearity. Thus, the following variables were included in the final model (for both genders): age at diagnosis, body mass index, presence of history of CHF, MI, stroke, chronic kidney disease (CKD: serum creatinine $\geq 2 \mathrm{mg} / \mathrm{dl}$ or regular hemodialysis), as well as treatment with CCBs. In addition to these previously-mentioned variables, the variables that were included in the final model for males were diabetes, malignancy, $\beta$ blockers and statins, and for females, hypertension, and LVEF. The model was proven to be highly significant ( $p$-value of the omnibus test for model coefficients $<0.001$ ). Eventually, age at diagnosis and the presence of CKD were significantly and independently associated with death, and CCB therapy was an independent inverse correlate of that event in both genders (Table 3 ). Additionally, the presence of diabetes (in males) and history of CHF (in females) were independently associated with higher mortality. 
Table 2 Clinical and Other Characteristics of Patients With IDD Who Survived Until January $1^{\text {st }} 2003$

\begin{tabular}{|c|c|c|c|}
\hline Variable & Male $(n=158)$ & Female $(n=114)$ & $p$ value \\
\hline \multicolumn{4}{|l|}{ Associated co-morbidities and their management } \\
\hline \multicolumn{4}{|l|}{ Cardiovascular risk factors/chronic medical illnesses } \\
\hline Age (years) & $68.5 \pm 8.7$ & $69.3 \pm 10.6$ & $N S$ \\
\hline Hypertension & $70(44.3 \%)$ & $55(48.2 \%)$ & $N S$ \\
\hline Diabetes mellitus & $46(29.1 \%)$ & $18(15.8 \%)$ & 0.016 \\
\hline Dyslipidemia & $25(15.8 \%)$ & $27(23.7 \%)$ & NS \\
\hline \multicolumn{4}{|l|}{ Body mass index } \\
\hline$\geq 25$ to $<30 \mathrm{~kg} / \mathrm{m}^{2}$ (overweight) & $35(22.2 \%)$ & $16(14.0 \%)$ & $N S$ \\
\hline$\geq 30 \mathrm{~kg} / \mathrm{m}^{2}$ (obesity) & $7(4.4 \%)$ & $2(1.8 \%)$ & NS \\
\hline \multicolumn{4}{|l|}{ Cardiac disorders } \\
\hline CHF hospitalization & $14(8.9 \%)$ & $13(11.4 \%)$ & $N S$ \\
\hline Angina pectoris & $10(6.3 \%)$ & $4(3.5 \%)$ & $N S$ \\
\hline Myocardial infarction & $20(12.7 \%)$ & $12(10.5 \%)$ & NS \\
\hline \multicolumn{4}{|l|}{ Arrhythmias } \\
\hline Atrial fibrillation & $61(38.6 \%)$ & $30(26.3 \%)$ & 0.046 \\
\hline Paroxysmal & $15(9.5 \%)$ & $10(8.8 \%)$ & NS \\
\hline Chronic & $46(29.1 \%)$ & $20(17.5 \%)$ & 0.031 \\
\hline Sustained ventricular tachyarrhythmia & $2(1.3 \%)$ & $0(0.0 \%)$ & NS \\
\hline Permanent cardiac pace maker & $9(5.7 \%)$ & $3(2.6 \%)$ & NS \\
\hline \multicolumn{4}{|l|}{ Cardiac surgery/intervention } \\
\hline Diagnostic coronary angiography & $50(31.7 \%)$ & $27(23.7 \%)$ & $N S$ \\
\hline Percutaneous coronary intervention & $11(7.0 \%)$ & $11(9.6 \%)$ & $N S$ \\
\hline Coronary artery bypass graft & $6(3.8 \%)$ & $3(2.4 \%)$ & $N S$ \\
\hline Cardiac valve replacement & $4(2.5 \%)$ & $0(0.0 \%)$ & $N S$ \\
\hline \multicolumn{4}{|l|}{ Neuropsychiatric disorders } \\
\hline Cerebral stroke & $37(23.4 \%)$ & $19(16.7 \%)$ & $N S$ \\
\hline Psychic illness & $9(5.7 \%)$ & $20(17.5 \%)$ & 0.004 \\
\hline \multicolumn{4}{|l|}{ Renal disorders } \\
\hline$C K D$ (serum creatinine $\geq 2 \mathrm{mg} / \mathrm{dl}$ or regular hemodialysis) & $15(9.5 \%)$ & $8(7.0 \%)$ & $N S$ \\
\hline \multicolumn{4}{|l|}{ Pulmonary disorders } \\
\hline Bronchial asthma & $4(2.5 \%)$ & $2(1.8 \%)$ & $N S$ \\
\hline Chronic obstructive pulmonary disease & $6(3.8 \%)$ & $0(0.0 \%)$ & $N S$ \\
\hline \multicolumn{4}{|l|}{ Level of daily activities and life style } \\
\hline Independently mobile & $151(95.6 \%)$ & $102(89.8 \%)$ & \\
\hline Wheel chair-dependent & $4(2.5 \%)$ & $4(3.5 \%)$ & $N S$ \\
\hline Bed-ridden & $3(1.9 \%)$ & $7(6.1 \%)$ & \\
\hline Solitary life & $12(7.6 \%)$ & $17(14.9 \%)$ & NS \\
\hline \multicolumn{4}{|l|}{ Educational background } \\
\hline$\leq 12$ years & $141(89.2 \%)$ & $101(88.6 \%)$ & \\
\hline$>12$ years & $17(10.8 \%)$ & $13(11.4 \%)$ & $N S$ \\
\hline Medications & & & \\
\hline Calcium-channel blockers & $69(43.7 \%)$ & $42(36.8 \%)$ & NS \\
\hline ACEIs or $A R B S$ & $68(43.0 \%)$ & $38(33.3 \%)$ & $N S$ \\
\hline Diuretics & $50(31.6 \%)$ & $26(22.8 \%)$ & NS \\
\hline Oral anticoagulants (warfarin) & $45(28.5 \%)$ & $30(26.3 \%)$ & $N S$ \\
\hline Acetyl salicylic acid (aspirin) & $39(24.7 \%)$ & $23(20.2 \%)$ & $N S$ \\
\hline$\beta$-adrenergic receptor blockers & $32(20.3 \%)$ & $17(14.9 \%)$ & NS \\
\hline Statins & $25(15.8 \%)$ & $17(14.9 \%)$ & $N S$ \\
\hline Nitrates & $20(12.7 \%)$ & $15(13.2 \%)$ & $N S$ \\
\hline Electrocardiographic data & & & \\
\hline Atrial fibrillation & $46(29.1 \%)$ & $20(17.5 \%)$ & 0.031 \\
\hline Left ventricular hypertrophy & $27(17.1 \%)$ & $18(15.8 \%)$ & $N S$ \\
\hline Right bundle branch block & $16(10.1 \%)$ & $7(6.1 \%)$ & NS \\
\hline Pathologic $Q$-wave & $17(10.8 \%)$ & $8(7.0 \%)$ & NS \\
\hline Echo data & & & \\
\hline Cardiac measurements & & & \\
\hline Ejection fraction $(\%)$ & $63.9 \pm 10.0$ & $68.5 \pm 8.4$ & $<0.001$ \\
\hline Left ventricular diastolic dimension ( $\mathrm{mm}$ ) & $50.6 \pm 6.1$ & $48.2 \pm 5.7$ & 0.001 \\
\hline Interventricular septum thickness (mm) & $11.1 \pm 2.9$ & $10.3 \pm 3.3$ & 0.035 \\
\hline Posterior wall thickness $(\mathrm{mm})$ & $10.5 \pm 2.2$ & $9.7 \pm 2.1$ & 0.003 \\
\hline$R W T$ & $0.42 \pm 0.10$ & $0.41 \pm 0.10$ & $N S$ \\
\hline $\operatorname{LVMI}\left(\mathrm{g} / \mathrm{m}^{2}\right)$ & $128.0 \pm 41.8$ & $123.7 \pm 51.0$ & NS \\
\hline Left atrial diameter $(\mathrm{mm})$ & $42.9 \pm 7.5$ & $40.8 \pm 8.0$ & 0.028 \\
\hline Left ventricular geometry & & & \\
\hline Normal & $53(33.5 \%)$ & $32(28.1 \%)$ & \\
\hline Eccentric hypertrophy & $37(23.4 \%)$ & $47(41.2 \%)$ & \\
\hline Concentric hypertrophy & $49(31.0 \%)$ & $32(28.1 \%)$ & 0.002 \\
\hline Concentric remodeling & $19(12.0 \%)$ & $3(2.6 \%)$ & \\
\hline Doppler data & & & \\
\hline E/A ratio & $1.3 \pm 0.5$ & $1.2 \pm 0.4$ & $N S$ \\
\hline Deceleration time $(\mathrm{ms})$ & $161.3 \pm 63.6$ & $150.1 \pm 48.6$ & $N S$ \\
\hline Deceleration time $<140 \mathrm{~ms}$ & $66(41.8 \%)$ & $56(49.1 \%)$ & NS \\
\hline
\end{tabular}




S/D ratio $<1$
AR-A duration
AR-A duration $>30 \mathrm{~ms}$
Isovoumetric relaxation time $(\mathrm{ms})$
Functional and prognostic indices
NYHA functional class
Class I
Class II-III
Class IV
Charlson co-morbidity index score
0
1
2
3
$\geq 4$

$S / D$ ratio $<1$

$A R-A$ duration

Isovoumetric relaxation time $(\mathrm{ms})$

NYHA functional class

Class I

Charlson co-morbidity index score

The Echo data shown are those obtained on initial recruitment of IDD.

Values are mean \pm standard deviation or number of subjects (\%).

Normal geometry: $R W T \leq 0.42$ and $L V M I \leq 115$ (95*) $\mathrm{g} / \mathrm{m}^{2}$.

Eccentric hypertrophy: $R W T \leq 0.42$ and $L V M I>115$ (95*) $\mathrm{g} / \mathrm{m}^{2}$.

Concentric hypertrophy: $R W T>0.42$ and $L V M I>115\left(95^{*}\right) \mathrm{g} / \mathrm{m}^{2}$.

Concentric remodeling: $R W T>0.42$ and $L V M I \leq 115\left(95^{*}\right) \mathrm{g} / \mathrm{m}^{2}$.

*Values for females.

IDD, isolated diastolic dysfunction; NS, non-significant; CHF, congestive heart failure; CKD, chronic kidney disease (serum creatinine $\geq 2 \mathrm{mg} / \mathrm{dl}$, or regular hemodialysis); ACEIs, angiotensin-converting enzyme inhibitors; ARBs, angiotensin-receptor blockers; Echo, echocardiographic; RWT, relative wall thickness; LVMI, left ventricular mass index; NYHA, New York Heart Association.

\section{Discussion}

The presence of IDD in asymptomatic individuals is an independent predictor for the development of CHF, so early identification and lifelong treatment of IDD is important for preventing progression of this serious condition? Not a few patients are presumed to be under treatment for IDD, but information is scarse in Japan. Our study design with its long recruitment period in an isolated area of the country was advantageous in delineating the epidemiological and clinical features of patients with IDD. We have made the following observations: (1) the number of outpatients with moderate to severe IDD is $0.5-1.5 \%$ of the elderly population; (2) the ratio of IDD to SD patients is larger in females (2.4) than in males (0.7); (3) the prevalence of hypertension and MI of this IDD cohort was similar to the estimates for IDD cohorts in the US, but lower than those of DHF cohorts in the US and Asia, whereas diabetes was more frequently observed in the present IDD cohort than in those from the US; (4) the LV mass index (LVMI) was high, but the LV geometry was diverse (with $31 \%$ of patients showing normal geometry; (5) although the majority of IDD patients had a rather low CCI score, their prognosis, even without a history of $\mathrm{CHF}$, was worse than that of general population; and (6) in both genders, higher age at diagnosis and the presence of CKD were independent predictors of mortality, whereas treatment with CCBs had a favorable survival benefit. Additionally, the presence of diabetes (in males) and history of CHF (in females) were indicators of bad prognosis.

In this study, the total numbers of IDD and SD patients increased with age, which is a common observation reported by some previous studies, $2,3 \mathrm{SD}$ was dominant in males, whereas IDD prevailed in female patients. We also found that the proportion of IDD patients in the general population was $0.9 \%$ for males and $0.5 \%$ for females in the age range of 45-84 years, based on the number of survivors in 2003 who were confirmed to have moderate to severe IDD. These estimates differ considerably from the prevalence rates reported by population-based studies in the US
Table 3 Independent Predictors of Mortality in Males and Females With IDD

\begin{tabular}{lcccc}
\hline \hline & $\beta$-coefficient & OR & $95 \%$ CI of OR & $p$ value \\
\hline Males & & & & \\
Age (years) & 0.132 & 1.141 & $1.080-1.207$ & $<0.001$ \\
CKD & 1.116 & 3.053 & $1.244-7.489$ & 0.015 \\
Diabetes & 1.042 & 2.836 & $1.271-6.326$ & 0.011 \\
CCBs & -0.169 & 0.184 & $0.068-0.499$ & 0.001 \\
Females & & & & \\
Age (years) & 0.110 & 1.117 & $1.036-1.204$ & 0.004 \\
CKD & 2.692 & 14.75 & $2.231-97.58$ & 0.005 \\
CHF & 1.884 & 6.578 & $1.284-33.71$ & 0.024 \\
CCBs & -2.756 & 0.064 & $0.009-0.441$ & 0.005 \\
\hline
\end{tabular}

OR, odds ratio; CI, confidence interval; CCBs, calcium channel blockers. Other abbreviations as in Table 2.

and Australia $(5.0-6.2 \%$ for males and $6.3-7.3 \%$ for females) $)^{2,3}$ In fact, detailed comparison would seem impossible for several reasons, such as ethnic, geodemographic, and methodological differences.

The literature indicates that patients with DHF are more likely to be females than those with SHF. In contrast to these US reports, males were dominant in our IDD cohort $(58 \%)$ as well in the DHF cohort reported by Tsutsui et al $(49-61 \%) !^{15}$ The reasons behind the male predominance remain unknown, but referral bias may explain this finding. Also, the literature indicates gender differences regarding consultation, examination, and treatment in cardiac patients ${ }^{16}$ which were unavoidable in our study owing to its hospital-based design. Thus, for comparing IDD and SD, it would be more appropriate to calculate the IDD/SD ratio after stratification by gender. This ratio was 0.7 for male and 2.4 for female patients, indicating more prevalence of IDD than SD among female patients who underwent Echo in this study. Therefore, we might suppose that a populationbased study of IDD that could carefully consider gender matching may demonstrate female predominance of IDD in the general population in Japan.

In a large population-based study conducted in Western 
communities, it has been reported that age, hypertension, diabetes, and MI were independent predictors of DD2,3 In Asian communities, these comorbidities are also associated with DHF. Tsutsui et al reported the clinical characteristics of Japanese patients with DHF (99 patients, average age 69 years) in Fukuoka ${ }^{15}$ In their study, the prevalence of hypertension, MI, and diabetes was $60 \%, 31 \%$, and $28 \%$, respectively. Similarly, Yip et al reported the prevalence of these comorbidities in Chinese patients with DHF (132 patients, average age of 73 years, $56 \%$ females) in Hong Kong; they found a prevalence of hypertension, MI, and diabetes of $57 \%, 14 \%$, and $35 \%$, respectively ${ }^{17}$ Compared with those reports, the prevalence of each of these comorbidities (hypertension, MI, and diabetes) was slightly lower in our IDD cohort ( $46 \%, 12 \%$, and $24 \%$, respectively). In addition, the majority $(90 \%)$ of our IDD cohort did not have a history of validated $\mathrm{CHF}$, in contrast with the Asian DHF cohorts in which most patients had such a history! 15,17 Therefore, these comorbidities may be significant risk factors for the advancement of IDD to DHF in Asian patients.

Regarding the Western populations, Vasan et al reported the clinical characteristics of 37 white patients admitted with DHF in Framingham (average age of 72 years, 65\% females) $!^{18}$ The prevalence of hypertension, MI, and diabetes was $75 \%, 24 \%$, and $14 \%$, respectively. In Olmsted County, Senni et al studied 59 white patients with DHF (average age of 78 years, $69 \%$ female) ${ }^{19}$ and reported a prevalence of hypertension (58\%) and MI (15\%) nearly similar to ours. In comparison with the estimates reported by studies of Asian populations (described in the previous paragraph), it could be noted that the prevalence of these comorbidities in Caucasian cohorts with DHF is closer to those in Asian communities. A similar prevalence of comorbidities in both DHF and IDD cohorts has been observed in Western communities?,18,19 Redfield et al conducted a population-based study in Olmsted County, and identified 118 residents with moderate IDD without a previous history of validated $\mathrm{CHF}^{2}$. The prevalence of hypertension, $\mathrm{MI}$, and diabetes in their cohort was $47 \%, 10 \%$, and $9 \%$, respectively, notably lower than the values found in some DHF cohorts in the US, 18,19 but rather closer to those found in our IDD cohort (except for diabetes).

The Doppler Echo selection criteria used in this study tended to include typically moderate to severe IDD patients; that is, DCT $<140 \mathrm{~ms}$, S/D ratio $<1$, and AR-A duration $>30 \mathrm{~ms}$ were observed in $45 \%, 51 \%$ and $29 \%$, respectively, of the patients, and the IRT was shortened. When divided by LVMI only, the LV geometry was hypertrophy in 54\% of males and $69 \%$ of females? On the other hand, when divided by LVMI and RWT (relative wall thickness), 5 the proportions of the 4 categories of LV geometry showed little differences, especially in males (Table 2). Thus, identifying IDD may be difficult without using a Doppler Echo technique, owing to the diverse nature of LV geometry.

It is not easy for conventional Doppler Echo techniques to evaluate the diastolic function in patients with chronic $\mathrm{AF}$, so we defined preserved systolic function associated with DCT $<140 \mathrm{~ms}$ as "restrictive DD", regardless of the presence of AF. There were several reasons for using this definition in our study. First, AF is common in patients with restrictive pathology, even though $12 \%$ of patients with normal sinus rhythm in this study had a documented history of AF (ie, paroxysmal AF). Second, an inverse relationship was reported between DCT and pulmonary capillary wedge pressure (PCWP) in patients with $\mathrm{AF}$, so a short DCT highly predicts elevated PCWP ${ }^{20}$ Furthermore, patients with and without AF who had a DCT $<130 \mathrm{~ms}$ showed a similarly poor prognosis. ${ }^{21}$ Third, this study aimed to evaluate the actual burden of IDD in the community, with its design targeting the high inclusion of clinically significant IDD subgroups (moderate to severe cases only, as the inclusion of mild cases will tend to underestimate the burden of IDD owing to the generally asymptomatic nature of the latter). Thus, we used conventional Echo-Doppler methodology that could be performed in most hospitals, using inclusion criteria that comprised undetermined and suspected IDD individuals (including AF cases) as well.

In comparison with the general population, our IDD patients (either with or without history of validated CHF) had poor survival, which may be related to the impact of IDD itself and/or other coexisting factors (such as diabetes, renal failure, cerebrovascular diseases, and dementia) that would predispose the patients to poor prognosis. The majority of patients (63\% of males and $64 \%$ of females) had CCI score $\leq 1$, but one-third of them had a score $\geq 2$, which made them a poor prognosis group according to some previous reports.22,23 Additionally, some of the comorbidities (diabetes, $\mathrm{CHF}$, and $\mathrm{CKD}$ ), which were also scored by the CCI, were shown to be independently associated with death in our cohort. In general, the higher the prevalence of poor prognostic comorbidities, the worse the patient's survival will be. Therefore, aging communities should pay attention to IDD and its associated comorbidities to reduce their future burden.

\section{Study Limitations}

First, because this study was hospital-based and not population-based, we may have missed latent IDD patients in the community who did not undergo Echo. Also, individuals with mild DD (impaired relaxation) were not included. Accordingly, estimation of the precise prevalence of the whole spectrum of IDD in the general population was not possible; however, we have shown the proportion of moderate to severe Echo-documented IDD. Moreover, the use of tissue Doppler for identifying IDD was not feasible. Nevertheless, we can suppose that diagnosing most patients with moderate to severe IDD could be easily done using conventional Echo-Doppler, and hence delineating the epidemiological and clinical features of these patients would closely mimic reality. Second, the limiting the study to Sado Island may restrict generalization of the results to other areas in Japan. Third, $72 \%$ of patients, especially the asymptomatic ones, did not undergo diagnostic coronary angiography. In this respect, it is known that noninvasive diagnostic processes have limitations in precisely classifying the disease etiology. Fourth, we tried to evaluate diastolic function in all patients; but this was not actually possible, especially in the elderly. Therefore, we might have underestimated the disease burden in the elderly patient category.

\section{Conclusions}

We have carried out the first enumeration survey of moderate to severe IDD in a Japanese community. The Sado Heart Failure Study is a highly inclusive hospital-based study that may be able to characterize patients with IDD in the community. Outpatients with moderate to severe IDD are progressively increasing with aging of the popultion. These patients often have multiple coexisting disorders, 
making the outcome of IDD poor, regardless of the presence of a CHF history. The disease characteristics and living conditions of patients should be taken into consideration when establishing preventive strategies for HF in the Japanese community.

\section{Acknowledgments}

This study was performed in collaboration with Dr Akira Hattori, Director, and Dr Takeshi Momotsu, Vice-director of Sado General Hospital; Drs Kenjiro Kikuchi, Takashi Ishikawa, Yoshio Kitami, and Keiko Saito of the Physiologic Laboratory; and the physicians of Sado Medical Society. Yuji Okura and Yukiko Ohno contributed equally to this work.

The study was funded by a Grant-in-Aid for Basic Research on Science and Technology $(C)$ : 'Comprehensive comparative evaluation of risk factors for hospitalization and death of patients with chronic heart failure in urban and rural regions' (17606003).

\section{References}

1. Braunwald E. Shattuck lecture: Cardiovascular medicine at the turn of the millennium: Triumphs, concerns, and opportunities. $N$ Engl $J$ Med 1997; 337: 1360-1369.

2. Redfield MM, Jacobsen SJ, Burnett JC, Mahoney DW, Bailey KR, Rodeheffer RJ. Burden of systolic and diastolic ventricular dysfunction in the community: Appreciating the scope of the heart failure epidemic. JAMA 2003; 289: 194-202.

3. Abhayaratna WP, Marwick TH, Smith WT, Becker NG. Characteristics of left ventricular diastolic dysfunction in the community: An echocardiographic survey. Heart 2006; 92: 1259-1264.

4. Department of Economic and Social Affairs, Population Division of United Nations. Percentage distribution of the population in selected age groups, by country, 2005 and 2050. In: World population prospects: The 2004 revision (highlights). New York: United Nations; 2005; 53-57.

5. Lang RM, Bierig M, Devereux RB, Flachskampf FA, Foster E, Pellikka PA, et al; American Society of Echocardiography's Nomenclature and Standards Committee; Task Force on Chamber Quantification; American College of Cardiology Echocardiography Committee; American Heart Association; European Association of Echocardiography, European Society of Cardiology. Recommendations for chamber quantification. Eur J Echocardiogr 2006; 7: 79-108.

6. McKee PA, Castelli WP, McNamara PM, Kannel WB. The natural history of congestive heart failure: The Framingham study. $N$ Engl $J$ Med 1971; 285: $1441-1446$.

7. Charlson ME, Pompei P, Ales KL, MacKenzie CR. A new method of classifying prognositic comorbidity in longitudinal studies: Development and validation. J Chronic Dis 1987; 40: 373-383.

8. Charlson M, Szatrowski TP, Peterson J, Gold J. Validation of a combined comorbidity index. J Clin Epidemiol 1994; 47: 1245-1251.

9. Chin MH, Goldman L. Correlates of early hospital readmission or death in patients with congestive heart failure. Am J Cardiol 1997; 79: $1640-1644$.

10. Tsutsui H, Tsuchihashi-Makaya M, Kinugawa S, Goto D, Takeshita A; JCARE-GENERAL Investigators. Characteristics and outcomes of patients with heart failure in general practices and hospitals Japanese cardiac registry of heart failure in general Practice (JCAREGENERAL). Circ J 2007; 71: 449-454.

11. Shiba N, Watanabe J, Shinozaki T, Koseki Y, Sakuma M, Kagaya Y, et al; CHART Investigators. Analysis of chronic heart failure registry in the Tohoku District: Third year follow up. Circ J 2004; 68: $427-$ 434.

12. Division of Statistics, Department of Policy and Planning, Niigata, Japan. Population estimates of Niigata prefecture, 2003. Niigata: The Department, 2003 (in Japanese).

13. Saunders BD, Trapp RG. Basic and clinical biostatistics, $2 \mathrm{~d}$ edn. Norwalk, CN: Appleton \& Lange; 1994.

14. Amitage P, Berry G. Statistical methods in medical research, 3rd edn. Oxford, UK: Blackwell Science; 2001.

15. Tsutsui H, Tsuchihashi M, Takeshita A. Mortality and readmission of hospitalized patients with congestive heart failure and preserved versus depressed systolic function. Am J Cardiol 2001; 88: 530-533.

16. Gan SC, Beaver SK, Houck PM, MacLehose RF, Lawson HW, Chan L. Treatment of acute myocardial infarction and 30-day mortality among women and men. N Engl J Med 2000; 343: 8-15.

17. Yip GW, Ho PP, Woo KS, Sanderson JE. Comparison of frequencies of left ventricular systolic and diastolic heart failure in Chinese living in Hong Kong. Am J Cardiol 1999; 84: 563-567.

18. Vasan RS, Larson MG, Benjamin EJ, Evans JC, Reiss CK, Levy D. Congestive heart failure in subjects with normal versus reduced left ventricular ejection fraction: Prevalence and mortality in a population-based cohort. J Am Coll Cardiol 1999; 33: 1948-1955.

19. Senni M, Tribouilloy CM, Rodeheffer RJ, Jacobsen SJ, Evans JM, Bailey KR, et al. Congestive heart failure in the community: A study of all incident cases in Olmsted County, Minnesota, in 1991. Circulation 1998; 98: 2282-2289.

20. Matsukida K, Kisanuki A, Toyonaga K, Murayama T, Nakashima H, Kumanohoso T, et al. Comparison of transthoracic Doppler echocardiography and natriuretic peptides in predicting mean pulmonary capillary wedge pressure in patients with chronic atrial fibrillation. $J$ Am Soc Echocardiogr 2001; 14: 1080-1087.

21. Hurrell DG, Oh JK, Mahoney DW, Miller FA Jr, Seward JB. Short deceleration time of mitral inflow E velocity: Prognostic implication with atrial fibrillation versus sinus rhythm. J Am Soc Echocardiogr 1998; 11: 450-457.

22. Sachdev M, Sun JL, Tsiatis AA, Nelson CL, Mark DB, Jollis JG. The prognostic importance of comorbidity for mortality in patients with stable coronary artery disease. J Am Coll Cardiol 2004; 43: $576-582$.

23. Jong P, Vowinckel E, Liu PP, Gong Y, Tu JV. Prognosis and determinants of survival in patients newly hospitalized for heart failure: A population-based study. Arch Intern Med 2002; 162: 1689-1694. 